\title{
Aneurysmal bone cyst
}

\section{A report of three cases}

\section{R. PUllaN, F. W. AlEXANDER, AND P. C. HALSE}

From the Department of Child Health, Newcastle General Hospital, and Royal Victoria Infirmary, Newcastle upon Tyne

SUMMARY Three children presenting with aneurysmal bone cysts are described. The first patient was 10 months old with a cyst of the scapula. The second was more typical but his cyst was treated initially as a malignant tumour. In the third child the second cervical vertebra was affected which posed considerable problems of management; it was treated by radiotherapy. Despite the problems all 3 children have made a good recovery.

An aneurysmal bone cyst is an uncommon benign lesion first defined and given the name by Jaffe and Lichtenstein (1942). It may be mistaken initially for a malignant tumour.

It presents most frequently in adolescents and young adults with pain or swelling; the spine and metaphyses of long bones are most often affected. $X$-ray shows a cystic lesion with expanded cortex and periosteal reaction. On exploration a haemorrhagic multilocular cyst is found with fibrous tissue and spicules of bone; histology shows a network of communicating blood-filled spaces and giant cells. Evacuation generally leads to a cure but there may be recurrences (Sharrard, 1971). Radiotherapy has been used but there is some suspicion of resulting sarcomatous change (Tillman et al., 1968). The cause is unknown but there is sometimes a history of previous trauma and there may be associated bone lesions (Aegerter and Kirkpatrick, 1968; Levy et al., 1975).

Three children recently presented to the Department of Child Health with aneurysmal bone cysts. These posed problems of diagnosis and management.

\section{Case studies}

Case 1. A 10-month-old baby boy presented in December 1975. He had been previously well. Four days before admission his mother noticed a lump on the right shoulder. It did not seem to be painful at first but when seen by us he was a little reluctant to move the arm and disliked the mass being palpated.

Received 20 February 1978
There was no history of trauma. Examination was normal apart from a firm mass in the right scapula measuring $3 \times 3 \mathrm{~cm}$. $X$-ray showed a swelling in the body of the scapula with cystic and sclerotic changes (Fig. 1).

Biopsy showed a cavity containing blood-stained serous fluid, necrotic material, and some loose bone. The cavity was curetted. Histology showed numerous dilated blood-filled spaces and multinucleate giant

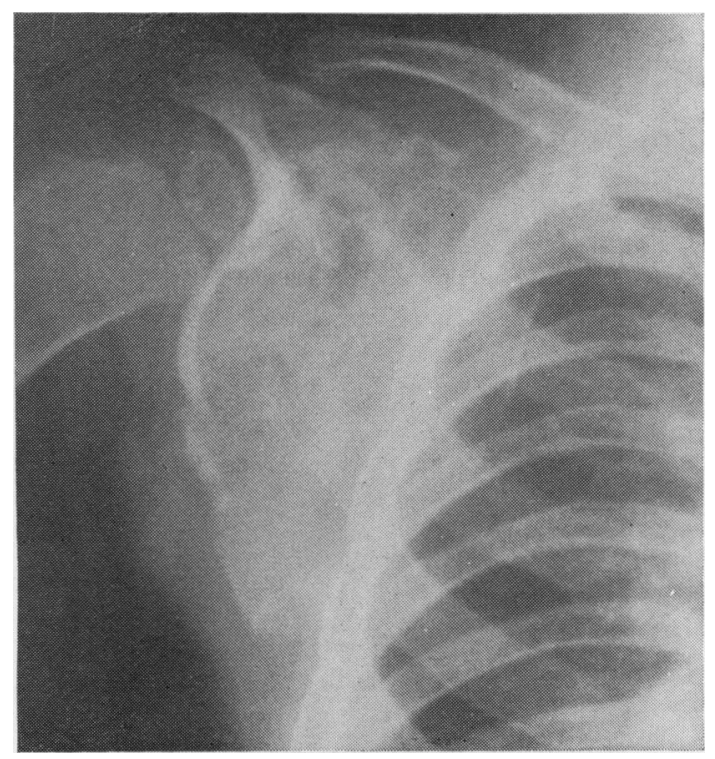

Fig. 1 Case 1. Cystic and sclerotic lesion in scapula. 
cells. There was no evidence of malignancy and the appearances were thought to be those of an aneurysmal bone cyst.

He made a good recovery and when last seen 10 months later the swelling had subsided. $X$-ray showed evidence of healing and was nearly back to normal.

Case 2. A 12-year-old boy presented in the casualty department of a neighbouring hospital in December 1975 after falling in snow. He complained of pain in the right upper arm. $X$-ray showed a spiral fracture of the midhumerus and a small bone cyst in the upper metaphysis. His arm was put in plaster and soon after removal of the plaster he was in a minor clash while playing football and returned again with pain in the right upper arm. $X$-ray now showed a fracture of the neck of the humerus through the cystic area. A further plaster was applied. He continued to complain of pain and on removal of the plaster the proximal half of the upper arm was found to be swollen and tender. $X$-ray showed extension of the cystic area with breaching of the cortex and periosteal elevation. A biopsy was performed and histology suggested an osteogenic sarcoma.

He was transferred to us for radiotherapy. Repeat $x$-rays (Fig. 2) looked more like an aneurysmal bone

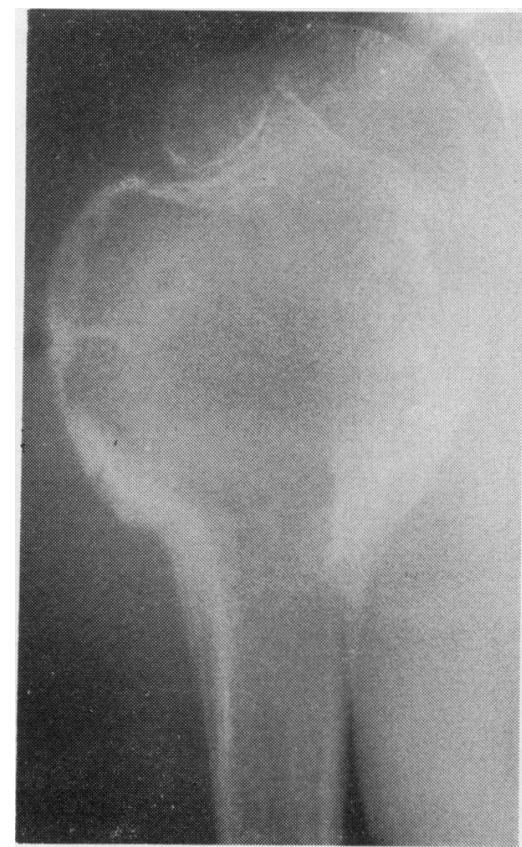

Fig. 2 Case 2. Cystic lesion in upper humerus. cyst and further opinion of the biopsy was sought. The histology was complicated by fracture callus and proliferation of fibroblastic tissue which contained a number of mitoses. This was thought to be more consistent with reactive fibrosis and granulation than with sarcoma, and a diagnosis of aneurysmal bone cyst was made. The course of radiotherapy was terminated. Curettage of the cyst was planned but delayed as the biopsy incision site became infected. The cyst was then found to be resolving and so nothing further was done. When seen 17 months later he was asymptomatic and $x$-ray showed progressive resolution.

Case 3. A 13-year-old boy presented in October 1976 with a 3-month history of stiff neck and subsequent swelling on the right side of his neck. There was no history of trauma. $X$-rays showed a destructive lesion mainly on the right side of the body of $\mathrm{C} 2$ but also on the neural arches of $\mathrm{C} 2$ and possibly C3 (Fig. 3). A biopsy was performed through the pharynx. Histology showed multinuclear giant cells in a not very vascular stroma, suggesting a giant cell tumour. In view of the $x$-rays and the boy's age, it was later thought more likely to be an aneurysmal bone cyst.

Resection or curettage was not thought practicable so he was treated with radiotherapy and had a total of 2000 rads over 3 weeks. He was nursed in a plaster bed. Subsequent $x$-rays showed recalcification of the vertebral body and also extensive calcification of the soft tissues both anterior to the first 3 cervical

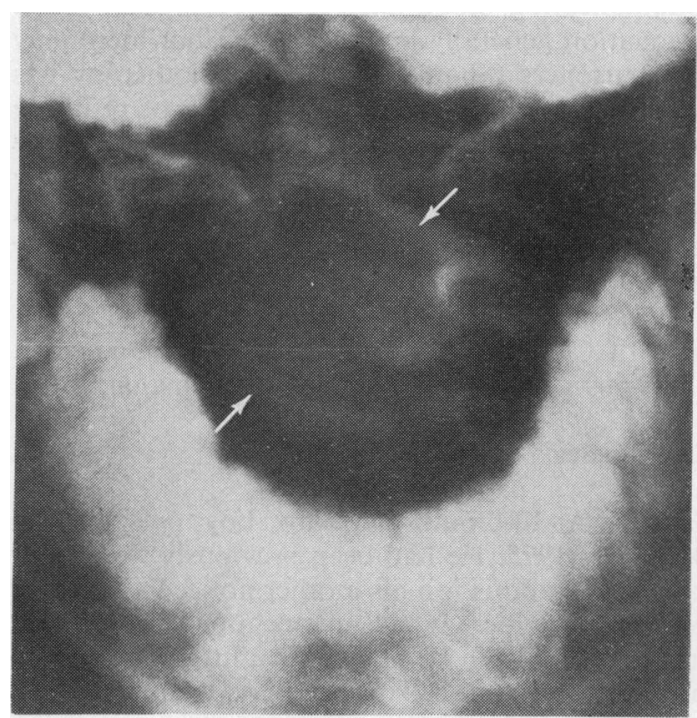

Fig. 3 Case 3. Destructive lesion in cervical spine. 
vertebrae and at the back of the neck around the laminae of the first 5 cervical vertebrae (Fig. 4). There was some forward subluxation of $\mathrm{C} 2$ on C3 but at no time did he have any neurological deficit. After 4 months he was mobilised in a Minerva plaster. This was removed after 7 weeks and he wore a collar for another 3 months. Nine months after radiotherapy he was asymptomatic, cervical spine movements were one-third normal, but pain free. $X$-ray showed progressive healing (Fig. 5).

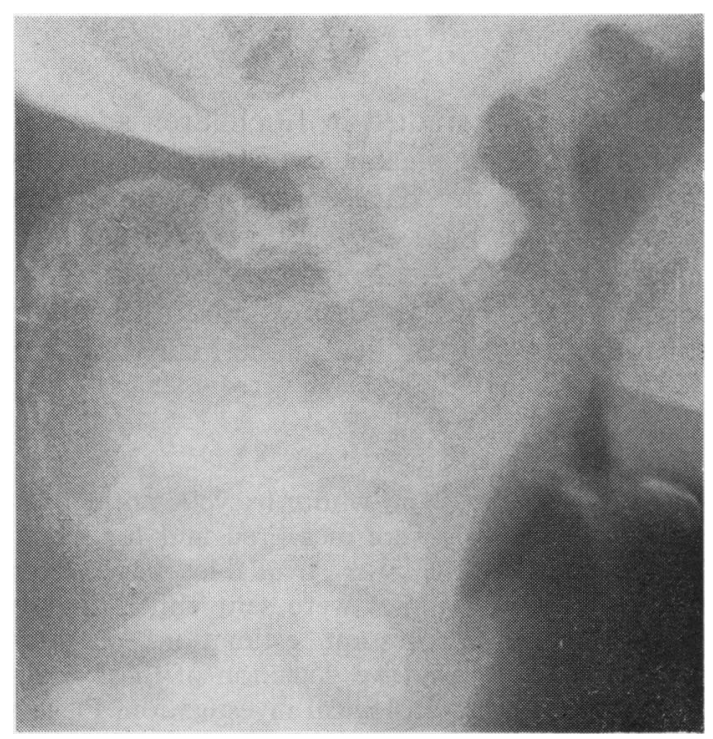

Fig. 4 Case 3. Six weeks after start of radiotherapy.

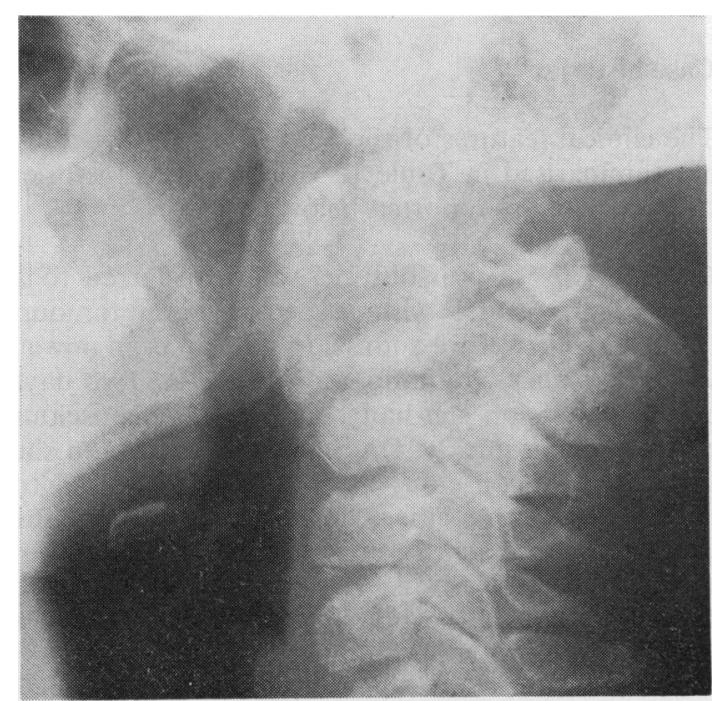

Fig. 5 Case 3. Nine months after radiotherapy.

\section{Discussion}

Cases 2 and 3 illustrate the more common types of presentation of aneurysmal bone cyst. They were adolescent and had a long bone and the spine involved. There was a clear history of trauma in Case 2, although not in Case 3. Case 1, however, is unusual in the age of presentation (10 months) and site (scapula). It is rare to find an aneurysmal bone cyst in infancy. Ginsburg (1974) described a neonate with an aneurysmal bone cyst after intrauterine fracture of the humerus. Levy et al. (1975) reported a series of 57 cases including a 5-month-old child with an associated unicameral bone cyst of the proximal tibia. In a series from the Mayo Clinic of 92 cases (Tillman et al., 1968) the youngest was 20 months, and in a series of 16 children from Paris (Rigault et al., 1972) the youngest was 3 years.

In all 3 children the lesion was first considered likely to be malignant. There was still uncertainty over the diagnosis after histology was obtained in Cases 2 and 3. Even after diagnosis was established, the treatment in Case 3 gave rise to considerable concern because of the site of the lesion. It was not considered safe to curette the cyst, so radiotherapy despite its small risks was used. There has been a satisfactory outcome in all 3 cases.

We thank Dr R. H. Jackson, Mr G. D. Stainsby, and Professor J. Stevens for permission to report their cases.

\section{References}

Aegerter, E. E., and Kirkpatrick, J. A., Jr (1968). Orthopedic Diseases, third edition, pp. 489-490. Saunders: Philadelphia.

Ginsburg, L. D. (1974). Congenital aneurysmal bone cyst. Radiology, 110, 175-176.

Jaffe, H. L., and Lichtenstein, L. (1942). Solitary unicameral bone cyst. Archives of Surgery, 44, 1004-1025.

Levy, W. M., Miller, A. S., Bonakdarpour, A., and Aegerter, E. (1975). Aneurysmal bone cyst secondary to other osseous lesions. American Journal of Clinical Pathology, 63, 1-8.

Rigault, P., Beneux, J., and Desvignes, P. (1972). Le kyste anévrysmal des os chez l'enfant. A propos de 16 cas. Annales de pédiatrie, 19, 223-234.

Sharrard, W. J. W. (1971). Paediatric Orthopaedics and Fractures, p. 753. Blackwell: Oxford.

Tillman. B. P., Dahlin, D. C., Lipscomb, P. R., and Stewart, J. R. (1968). Aneurysmal bone cyst: an analysis of 95 cases. Mayo Clinic Proceedings, 43, 478-495.

Correspondence to Dr C. R. Pullan, Department of Virology, Royal Victoria Infirmary, Queen Victoria Road, Newcastle upon Tyne NE1 4LP. 